\title{
"Seizing" the opportunity to understand antifibrinolytic drugs
}

\author{
Irene Lecker, BSc $\cdot$ Beverley A. Orser, MD, PhD • \\ C. David Mazer, MD
}

Received: 14 October 2011/ Accepted: 19 October 2011/Published online: 4 November 2011

(C) Canadian Anesthesiologists' Society 2011

\begin{abstract}
Antifibrinolytic drugs have been used for decades primarily due to their capacity to decrease blood loss and reduce the need for allogeneic transfusion in patients undergoing cardiac or noncardiac surgery. The three main antifibrinolytic drugs are aprotinin, which was first used in Germany in the late $1950 \mathrm{~s}$; aminocaproic acid, which was approved by the Food and Drug Administration in 1964; and tranexamic acid (TXA), which was made available in 1986. It is remarkable that despite widespread use of these drugs over several decades, there is still no consensus regarding their optimal dose and dosing regimen. The need to resolve this debate is becoming increasingly urgent, as the clinical use of antifibrinolytics is expected to rise due to the increasing number of complex surgeries being performed and the expanded use beyond cardiac surgery. Specifically, the use of TXA in noncardiac surgery is likely to increase
\end{abstract}

C. David Mazer has received research funding and/or honoraria from NovoNordisk, Cubist, Bayer, The Medicines Company, Oxygen Biotherapeutics, Hemcon and AstraZeneca

I. Lecker, BSc

Department of Physiology, University of Toronto,

Toronto, ON, Canada

B. A. Orser, MD, PhD - C. D. Mazer, MD

Departments of Anesthesia and Physiology,

University of Toronto, Toronto, ON, Canada

B. A. Orser, $\mathrm{MD}, \mathrm{PhD}$

Department of Anesthesia, Sunnybrook Health Sciences Centre,

Toronto, ON, Canada

C. D. Mazer, MD ( $\square)$

Department of Anesthesia, Keenan Research Center in the

Li Ka Shing Knowledge Institute of St. Michael's Hospital,

30 Bond Street, Toronto, ON M5B 1W8, Canada

e-mail: mazerd@smh.ca following the recent demonstration that this drug reduces mortality in trauma patients. ${ }^{1}$ Moreover, all three agents are associated with significant adverse effects. Tranexamic acid has been reported to be associated with an increased incidence of postoperative seizures, ${ }^{2}$ whereas aprotinin has been associated with increases in renal dysfunction and mortality. ${ }^{3}$ Thus, serious questions remain about the relative efficacy and safety of antifibrinolytic drugs.

In this issue of the Journal, Manji et al. ${ }^{4}$ address a pertinent safety concern related to the use of antifibrinolytic drugs: the incidence of postoperative seizures in patients who have undergone cardiac surgery. The aim of their study was to identify risk factors for seizures following cardiac surgery. Manji et al. performed a retrospective analysis of consecutive patients who underwent cardiac surgery between April 2003 and December 2009. Despite the limitations of retrospective analyses (including, in this instance, missing data for $5-10 \%$ of patients and lack of specific monitoring for seizures), the authors identified seven important factors that were significantly associated with seizures: exposure to TXA, APACHE II score $>20$, preoperative cardiac arrest, preoperative neurological disease, open chamber surgery, cardiopulmonary bypass time $>150 \mathrm{~min}$, and previous cardiac surgery. All of these risk factors, other than TXA exposure, indicate that the incidence of seizures is strongly associated with the "sickness" of the patient.

The authors also found a relationship between changes in TXA dosing and incidence of seizures over time. More specifically, patients whose estimated TXA dose was between 1.4 and $1.7 \mathrm{~g}$ (during the period 2003-2006) had a seizure rate of $0.0-0.3 \%$, whereas those whose TXA dose was between 5.1 and $5.8 \mathrm{~g}$ (over the period 2007-2009) had a seizure rate of 1.2-1.8\%. Reduction of the mean TXA dose to $3.1 \mathrm{~g}$ (in 2010) was associated with a concurrent decrease in 
the incidence of seizures, from 1.8-0.8\%. This dose-response relationship suggests that TXA may be a modifiable risk factor for seizures and thus represents an important contribution to the debate about the appropriate dose of TXA for patients undergoing cardiac surgery.

Although the potential proconvulsant properties of TXA have been previously reported, ${ }^{5}$ the findings reported by Manji et al. showcase two important concerns: first, the need to determine the optimal antifibrinolytic dose for cardiac procedures, and second, the need to understand the molecular mechanism by which antifibrinolytic agents could cause postoperative seizures in cardiac patients.

The lack of consensus regarding optimal antifibrinolytic therapy is reflected in conflicting recommendations regarding the use of aprotinin. The 2011 joint guidelines of the Society of Thoracic Surgeons and the Society of Cardiovascular Anesthesiologists state that aprotinin is not indicated for "routine blood conservation because the risks of adverse events outweigh the benefits."(Class III recommendation, Level A evidence). ${ }^{6}$ In addition, the 2011 Cochrane review of antifibrinolytic drugs concluded that although "aprotinin appears to be slightly more effective than the lysine analogues in reducing blood loss and the receipt of blood transfusion... head to head comparisons show a lower risk of death with lysine analogues when compared with aprotinin". 7 In contrast, Health Canada recently concluded that "the benefits of Trasylol [aprotinin] outweigh the risks when Trasylol is used as authorized by Health Canada." 8

The disagreement regarding the most effective dosing regimen, including optimal dose, of these drugs is just as intense. A wide variety of dosing regimens have been recommended, including bolus administration with or without additional infusions, fixed doses, weight-based dosing, priming of the cardiopulmonary bypass pump, and topical application, but none has emerged as clearly superior to the others. A commonly used TXA dosing regimen, originally employed by the Blood Conservation Using Antifibrinolytics in a Randomized Trial (BART), targets a plasma TXA concentration of about $800 \mu \mathrm{M}$ (thought to be achieved with a $30 \mathrm{mg} \cdot \mathrm{kg}^{-1}$ bolus dose, $2 \mathrm{mg} \cdot \mathrm{kg}^{-1}$ added to the pump prime, and $16 \mathrm{mg} \cdot \mathrm{kg}^{-1} \cdot \mathrm{hr}^{-1}$ for the duration of the operation). ${ }^{3}$ However, a TXA concentration of $127 \mu \mathrm{M}$ is sufficient to provide $>90 \%$ inhibition of tissue activators of fibrinolysis. ${ }^{9}$ Therefore, the plasma concentrations targeted with the BART regimen are roughly six times higher than is necessary for some antifibrinolytic activity. Though Manji et al. demonstrate that a lower TXA dose $\left(45 \mathrm{mg} \cdot \mathrm{kg}^{-1}\right)$ was associated with a lower frequency of seizures, it is noteworthy that a recent randomized trial using two different doses of TXA reported greater efficacy, fewer seizures and lower mortality with the higher BART dosage. ${ }^{10}$ Thus, the determination of the optimal dose and dosing regimen must balance the need for antifibrinolytic activity and the necessity of minimizing serious adverse effects, such as postoperative seizures.

Seizure following cardiac surgery represents a potentially serious complication that can be associated with prolongation of recovery and increases in morbidity and mortality. ${ }^{11}$ Seizures can seriously increase cardiovascular strain and metabolic demands. ${ }^{11}$ In addition, a diagnosis of "epilepsy" could negatively affect the patient's quality of life and independence. ${ }^{12}$ However, whether an isolated single postoperative seizure in the absence of evidence of brain injury significantly worsens outcome remains to be determined. The frequency of seizures in cardiac patients is generally low $(0.5 \%),{ }^{13}$ but increases with the administration of TXA, to a level ranging from 2.7-7.6\%, with noncoronary artery bypass grafting or open ventricle procedures having the highest incidence. ${ }^{14,15}$

Unfortunately, many questions still exist about the underlying mechanism responsible for TXA-associated postoperative seizures. They typically occur in the intensive care unit, ${ }^{2}$ long after the administration of TXA has been stopped, at a point when theoretically the serum and brain levels of the drug should be declining. One possible explanation for this paradoxical observation is that TXA builds up in the brain during the surgery, especially in patients with disruption of the blood-brain barrier. Alternatively, there may be a masking effect whereby anesthetics administered during the surgery inhibit proconvulsant effects of TXA.

Tranexamic acid-mediated seizures are traditionally explained by two mechanisms. The first of these is the cerebral ischemia hypothesis, derived from the concept that TXA could generate vasospasm or promote thrombosis and thus reduce cerebral blood flow. ${ }^{16}$ The second potential mechanism through which TXA might induce seizures is the inhibition of $\gamma$-aminobutyric acid type $\mathrm{A}$ receptors $\left(\mathrm{GABA}_{\mathrm{A}} \mathrm{Rs}\right)$. $\mathrm{GABA}_{\mathrm{A}} \mathrm{Rs}$ are crucial for maintaining inhibitory neurotransmission, and a decrease in $\mathrm{GABA}_{\mathrm{A}} \mathrm{R}$ activity is generally associated with an increased probability of seizure. Previous authors have demonstrated that TXA is a competitive antagonist of $\mathrm{GABA}_{\mathrm{A}} \mathrm{Rs},{ }^{17}$ estimating the mean inhibitory half maximal concentration $\left(\mathrm{IC}_{50}\right) \pm$ standard deviation at $7.1 \pm 3.1 \mathrm{mM} \quad\left(1.12 \pm 0.49 \mathrm{mg} \cdot \mathrm{mL}^{-1}\right) .{ }^{17}$ According to these estimations, the $\mathrm{GABA}_{\mathrm{A}} \mathrm{Rs}$ are probably insensitive to TXA inhibition, given that the concentration of TXA in the cerebrospinal fluid of patients after surgery is estimated to be significantly lower - in the range of 0.7$1.3 \mathrm{mM}\left(0.1-0.2 \mathrm{mg} \cdot \mathrm{mL}^{-1}\right){ }^{9}$ This suggests that one or more other receptors may be involved in TXA-associated seizures. Further research is required to better understand the molecular mechanisms underlying these seizures, to document the TXA concentrations in the blood and CSF, and to identify potential treatment or prevention strategies. 
As the reliance on perioperative antifibrinolytics increases, there is increasing urgency to resolve the debate over optimal dosage and to better understand the mechanism of action of these drugs. The recent decision by Health Canada to allow resumption of marketing of aprotinin in Canada raises many questions related to the efficacy, safety, optimal dosing and cost-effectiveness of antifibrinloytic agents. Treatment protocols should not be based on inadequate evidence, anecdotal experience, marketing, lobbying or personal bias. Rather, we should seize the opportunity to undertake rigorous studies that will provide definitive answers to these questions. The work by Manji et al. is a step in the right direction. Our patients expect and deserve no less from us.

\section{Saisir la chance de comprendre les antifibrinolytiques}

Les antifibrinolytiques sont utilisés depuis plusieurs décennies, essentiellement en raison de leur capacité à limiter les pertes sanguines et diminuer le recours à des transfusions de sang allogène chez des patients subissant une chirurgie cardiaque ou non cardiaque. Les trois principaux médicaments antifibrinolytiques sont l'aprotonine, qui a d'abord été utilisée en Allemagne à la fin des années 1950; l'acide aminocaproïque, qui a été approuvé par la Food and Drug Administration (FDA) en 1964; et l'acide tranexamique (TXA), qui est disponible depuis 1986. Néanmoins, en dépit de l'utilisation généralisée de ces médicaments pendant plusieurs décennies, on constate avec étonnement qu'il n'existe toujours pas de consensus sur leurs doses optimales et leurs posologies. Résoudre cette question devient une nécessité de plus en plus pressante, car on peut s'attendre à voir l'utilisation clinique des antifibrinolytiques augmenter en raison, d'une part, du nombre croissant des interventions chirurgicales complexes qui sont réalisées et, d'autre part, de l'élargissement de leurs indications au-delà de la chirurgie cardiaque. Plus précisément, l'utilisation du TXA en chirurgie non cardiaque va probablement croître après la récente démonstration de la réduction de la mortalité chez les patients traumatisés grâce à ce médicament. ${ }^{1}$ De plus, ces trois molécules sont associées à des effets indésirables significatifs. Une augmentation de l'incidence des crises convulsives postopératoires a été décrite en association avec le $\mathrm{TXA}^{2}$ tandis que l'aprotonine était associée à une augmentation d'insuffisance rénale et de mortalité. ${ }^{3}$ Il reste donc des questions sérieuses concernant l'efficacité relative et l'innocuité des antifibrinolytiques.
Dans ce numéro du Journal, Manji et coll. ${ }^{4}$ abordent une préoccupation pertinente concernant l'innocuité de l'utilisation des antifibrinolytiques : l'incidence des crises convulsives postopératoires chez les patients ayant subi une chirurgie cardiaque. Le but de leur étude était d'identifier les facteurs de risque de convulsions après ce type de chirurgie. Manji et coll. ont mené une analyse rétrospective de patients consécutifs ayant subi une chirurgie cardiaque entre avril 2003 et décembre 2009. Malgré les limites liées aux analyses rétrospectives (incluant, dans ce cas-ci, des données manquantes pour $5 \%$ à $10 \%$ des patients et un manque de suivi spécifique des crises convulsives), les auteurs ont identifié sept facteurs importants associés de façon significative aux crises convulsives: l'exposition au TXA, un score APACHE II $>20$, un arrêt cardiaque préopératoire, une maladie neurologique préopératoire, une chirurgie à coeur ouvert, une durée de circulation extracorporelle $>150 \mathrm{~min}$ et un antécédent de chirurgie cardiaque. Tous ces facteurs de risque, outre l'exposition au TXA, indiquent que l'incidence des crises convulsives est fortement associée au «mauvais état de santé » du patient.

Les auteurs ont également trouvé une relation entre les changements de posologie du TXA et l'incidence des crises convulsives au fil des années. En particulier, la fréquence des convulsions chez les patients ayant reçu une dose de TXA comprise entre 1,4 et $1,7 \mathrm{~g}$ (au cours de la période 2003-2006) était de 0,0 à 0,3\%, alors qu'elle était de 1,2 à $1,8 \%$ chez les patients dont la dose de TXA était comprise entre 5,1 et 5,8 $\mathrm{g}$ (période 2007-2009). La réduction de la dose moyenne de TXA à $3,1 \mathrm{~g}$ (en 2010) a été associée à une baisse simultanée de l'incidence des convulsions: de 1,8 à $0,8 \%$. Cette relation dose-effet suggère que le TXA peut être un facteur de risque modifiable de crises convulsives; cela constitue une contribution importante au débat sur la dose de TXA qui est indiquée chez des patients subissant une chirurgie cardiaque.

Même si les propriétés proconvulsivantes du TXA ont déjà été décrites, ${ }^{5}$ les résultats fournis par Manji et coll. mettent en évidence deux préoccupations importantes: premièrement, la nécessité de déterminer la dose optimale d'antifibrinolytique pour les interventions cardiaques et, deuxièmement, la nécessité de comprendre le mécanisme moléculaire par lequel les antifibrinolytiques sont susceptibles de provoquer des crises convulsives chez les patients subissant une chirurgie cardiaque.

L'absence de consensus sur le traitement antifibrinolytique optimal apparaît dans les recommandations contradictoires concernant l'utilisation de l'aprotinine. Les lignes directrices conjointes 2011 de la Society of Thoracic Surgeons et de la Society of Cardiovascular Anesthesiologists mentionnent que l'aprotinine n'est pas indiquée pour une « conservation du sang sur une base habituelle, car les risques d'événements 
indésirables dépassent largement ses avantages » (Recommandation de catégorie III et preuve de niveau A). ${ }^{6} \mathrm{De}$ plus, une étude Cochrane de 2011 sur les antifibrinolytiques est parvenue à la conclusion suivante: « Bien que l'aprotinine paraisse légèrement plus efficace que les analogues de la lysine pour la réduction des pertes de sang et du besoin transfusionnel, des comparaisons bilatérales ont montré un risque de décès moindre avec les analogues de la lysine qu'avec l'aprotinine $» .^{7}$ En revanche, Santé Canada a récemment conclu que les « avantages du Trasylol (aprotinine) dépassent largement les risques quand le Trasylol est utilisé comme autorisé par Santé Canada ${ }^{8}{ }^{8}$

Le désaccord concernant le régime posologique le plus efficace avec le TXA, y compris sa dose optimale, n'en est que plus intense. Des posologies très variées ont été recommandées, y compris une administration en bolus avec ou sans perfusions associées, des doses fixes, des doses en fonction $\mathrm{du}$ poids, un amorçage de la circulation extracorporelle (CEC), et une application topique, mais aucune ne s'est avérée clairement supérieure aux autres. Un régime posologique souvent employé, utilisé initialement dans l'essai randomisé BART (Blood Conservation Using Antifibrinolytics - Conservation du sang au moyen des antifibrinolytiques), vise une concentration plasmatique de TXA d'environ $800 \mu \mathrm{M}$ (que l'on estimait pouvoir être obtenue avec un bolus de $30 \mathrm{mg} \cdot \mathrm{kg}^{-1}, 2 \mathrm{mg} \cdot \mathrm{kg}^{-1}$ ajoutés à l'amorçage de la CEC et $16 \mathrm{mg} \cdot \mathrm{kg}^{-1} \cdot \mathrm{h}^{-1}$ pour la durée de l'intervention). ${ }^{3}$ Cependant, une concentration de TXA de $127 \mu \mathrm{M}$ est suffisante pour assurer plus de $90 \%$ d'inhibition des activateurs tissulaires de la fibrinolyse. ${ }^{9}$ Les concentrations plasmatiques ciblées avec le protocole BART sont donc environ six fois plus élevées que ce qui est nécessaire pour obtenir une activité antifibrinolytique. Bien que Manji et coll. aient démontré qu'une dose plus faible de TXA (45 mg. $\mathrm{kg}^{-1}$ ) est associée à une fréquence moindre de crises convulsives, il est intéressant de noter qu'un essai randomisé récent utilisant deux doses différentes de TXA a décrit une plus grande efficacité, moins de crises convulsives et une mortalité plus basse avec la posologie BART la plus élevée. ${ }^{10}$ Ainsi, la dose optimale et le régime posologique doivent être déterminées en trouvant un juste milieu entre le besoin d'une activité fibrinolytique et la nécessité de minimiser les effets indésirables graves postopératoires tels que les crises convulsives.

Les crises convulsives après chirurgie cardiaque représentent une complication potentiellement grave qui peut être associée à une convalescence plus longue et à une augmentation de la morbidité et de la mortalité. ${ }^{11}$ Les crises convulsives peuvent augmenter sérieusement le travail du myocarde et les besoins métaboliques. ${ }^{11}$ De plus, un diagnostic de « crise convulsive » peut avoir un impact négatif sur la qualité de vie et l'indépendance du patient. ${ }^{12}$ Il reste cependant à déterminer si une crise convulsive postopératoire unique et isolée en l'absence de signes de lésion cérébrale aggrave l'évolution de façon significative. La fréquence des crises convulsives chez les patients cardiaques est habituellement faible $(0,5 \%),{ }^{13}$ mais augmente avec l'administration de TXA pour atteindre une incidence allant de $2,7 \%$ à 7,6 \%, les interventions sans pontage coronarien ou à ventricule ouvert ayant les incidences les plus élevées. ${ }^{14,15}$

Il reste malheureusement de nombreuses questions concernant le mécanisme sous-jacent responsable des crises convulsives postoperatoires associées au TXA. Ces crises surviennent habituellement à l'unité des soins intensifs, ${ }^{2}$ longtemps après la fin de l'administration de TXA, à un stade où, théoriquement, les concentrations sériques et cérébrales du médicament devraient diminuer. Une explication possible de cette observation paradoxale est que le TXA s'accumule dans le cerveau pendant la chirurgie, en particulier chez des patients ayant une rupture de la barrière hémato-encéphalique. Il pourrait aussi y avoir un effet de masquage, les anesthésiques administrés au cours de l'intervention inhibant les effets proconvulsivants du TXA.

Les crises convulsives médiées par le TXA sont traditionnellement expliquées par deux mécanismes. Le premier repose sur l'hypothèse d'une ischémie cérébrale sur la base du concept que le TXA pourrait provoquer un vasospasme ou promouvoir une thrombose, réduisant ainsi le débit sanguin cérébral. ${ }^{16}$ Le deuxième mécanisme potentiel par lequel le TXA pourrait induire des crises convulsives est l'inhibition des récepteurs de l'acide $\gamma$-aminobutyrique de type $\mathrm{A}\left(\mathrm{GABA}_{\mathrm{A}} \mathrm{R}\right)$. Les $\mathrm{GABA}_{\mathrm{A}} \mathrm{R}$ sont essentiels au maintien de la neurotransmission inhibitrice et une diminution de leur activité est généralement associée à une plus grande probabilité de crise convulsive. Des auteurs ont montré précédemment que le TXA est un antagoniste compétitif des $\mathrm{GABA}_{\mathrm{A}} \mathrm{R},{ }^{17}$ estimant la concentration maximale inhibitrice $50\left(\mathrm{IC}_{50}\right) \pm$ écart-type à $7,1 \pm 3,1 \mathrm{mM}\left(1,12 \pm 0,49 \mathrm{mg} \cdot \mathrm{mL}^{-.1}\right) .{ }^{17}$ Selon ces estimations, les $\mathrm{GABA}_{\mathrm{A}} \mathrm{R}$ ne sont probablement pas sensibles à l'inhibition par le TXA compte tenu du fait que l'on estime que la concentration de TXA dans le liquide céphalorachidien des patients après chirurgie est significativement plus basse (dans une fourchette de 0,7 à 1,3 mM, soit $\left.0,1-0,2 \mathrm{mg} \cdot \mathrm{mL}^{-1}\right){ }^{9}$ Ceci suggère qu'un ou plusieurs autres récepteurs peuvent être impliqués dans les crises convulsives associée au TXA. Des recherches supplémentaires sont nécessaires pour mieux comprendre les mécanismes moléculaires sous-tendant ces crises convulsives, pour documenter les concentrations de TXA dans le sang et le LCR, et pour identifier un traitement potentiel ou des stratégies de prévention.

Considérant que le recours aux antifibrinolytiques augmente en période périopératoire, il devient de plus en 
plus urgent de résoudre cette question d'une posologie optimale et de mieux comprendre le mécanisme d'action de ces médicaments. La décision récente de Santé Canada d'autoriser la reprise de la commercialisation de l'aprotinine au Canada soulève de nombreuses questions à propos de l'efficacité, l'innocuité, la posologie optimale et le rapport coût-efficacité des antifibrinolytiques. Les protocoles thérapeutiques ne doivent pas s'appuyer sur des preuves inappropriées, une expérience anecdotique, du marketing, du lobbying ou des biais personnels. Nous devrions plutôt saisir cette opportunité pour entreprendre des études rigoureuses qui nous fourniront des réponses définitives à ces questions. À ce titre, le travail de Manji et coll. est un pas dans la bonne direction. Nos patients n'en attendent et n'en méritent pas moins de notre part.

Funding I. Lecker is supported by a Scholarship from the Savoy Foundation. B.A.O. is supported by an operating grant from the Canadian Institutes of Health Research (MOP 38028), and a Canadian Anesthesiologists' Society grant and a Canada Research Chair in Anaesthesia.

\section{References}

1. CRASH-2 Trial Collaborators, Shakur H, Roberts I, Bautista R, et al. Effects of tranexamic acid on death, vascular occlusive events, and blood transfusion in trauma patients with significant haemorrhage (CRASH-2): a randomised, placebo-controlled trial. Lancet 2010; 376: 23-32.

2. Murkin JM, Falter F, Granton J, Young B, Burt C, Chu M. Highdose tranexamic acid is associated with nonischemic clinical seizures in cardiac surgical patients. Anesth Analg 2010; 110: 350-3.

3. Fergusson DA, Hebert PC, Mazer CD, et al. A comparison of aprotinin and lysine analogues in high-risk cardiac surgery. $\mathrm{N}$ Engl J Med 2008; 358: 2319-31.

4. Manji RA, Grocott HP, Leake J, et al. Seizures following cardiac surgery: the impact of tranexamic acid and other risk factors. Can J Anesth 2012; 59: this issue. DOI:10.1007/s12630-011-9618-z.
5. Kaabachi $O$, Eddhif M, Rais $K$, Zaabar MA. Inadvertent intrathecal injection of tranexamic acid. Saudi J Anaesth 2011; 5: 90-2.

6. Society of Thoracic Surgeons Blood Conservation Guideline Task Force, Ferraris VA, Brown JR, Despotis GJ, et al. 2011 update to the Society of Thoracic Surgeons and the Society of Cardiovascular Anesthesiologists blood conservation clinical practice guidelines. Ann Thorac Surg 2011; 91: 944-82.

7. Henry DA, Carless PA, Moxey AJ, et al. Anti-fibrinolytic use for minimising perioperative allogeneic blood transfusion. Cochrane Database Syst Rev 2011; 3: CD001886.

8. Health Canada. Health Canada Decision on Trasylol (aprotinin). Information Update September 2011. Available from URl: http:// www.hc-sc.gc.ca/ahc-asc/media/advisories-avis/2011/2011_124eng.php. (accessed October, 2011).

9. Dowd NP, Karski JM, Cheng DC, et al. Pharmacokinetics of tranexamic acid during cardiopulmonary bypass. Anesthesiology 2002; 97: 390-9.

10. Bokesch PM, Szabo G, Wojdyga R, et al. A phase 2 prospective, randomized, double-blind trial comparing the effects of tranexamic acid with ecallantide on blood loss from high-risk cardiac surgery with cardiopulmonary bypass (CONSERV-2 Trial). J Thorac Cardiovasc Surg 2011; doi: 10.1016/j.jtcvs.2011.06.001.

11. Hunter GR, Young GB. Seizures after cardiac surgery. J Cardiothorac Vasc Anesth 2011; 25: 299-305.

12. Laccheo I, Ablah E, Heinrichs $R$, Sadler T, Baade L, Liow $K$. Assessment of quality of life among the elderly with epilepsy. Epilepsy Behav 2008; 12: 257-61.

13. Roach GW, Kanchuger M, Mangano CM, et al. Adverse cerebral outcomes after coronary bypass surgery. Multicenter Study of Perioperative Ischemia Research Group and the Ischemia Research and Education Foundation Investigators. N Engl J Med 1996; 335: 1857-63.

14. Sander M, Spies CD, Martiny V, Rosenthal C, Wernecke KD, von Heymann $C$. Mortality associated with administration of highdose tranexamic acid and aprotinin in primary open-heart procedures: a retrospective analysis. Crit Care 2010; 14: R148.

15. Martin K, Knorr J, Breuer T, et al. Seizures after open heart surgery: comparison of epsilon-aminocaproic acid and tranexamic acid. J Cardiothorac Vasc Anesth 2011; 25: 20-5.

16. Iplikcioglu AC, Berkman MZ. The effect of short-term antifibrinolytic therapy on experimental vasospasm. Surg Neurol 2003; 59: 10-6. discussion 16-7.

17. Furtmuller $R$, Schlag $M G$, Berger $M$, et al. Tranexamic acid, a widely used antifibrinolytic agent, causes convulsions by a gamma-aminobutyric acid(A) receptor antagonistic effect. J Pharmacol Exp Ther 2002; 301: 168-73. 